\title{
On the Solutions of Four Second-Order Nonlinear Difference Equations
}

\author{
İnci Okumuş, Yüksel Soykan
}

\begin{abstract}
Zonguldak Bülent Ecevit University, Department of Mathematics, Art and Science Faculty, 67100, Zonguldak, Turkey e-mail: inci_okumus_90@hotmail.com (corresponding author) yuksel_soykan@hotmail.com
\end{abstract}

\section{Abstract}

This paper deals with the form, the stability character, the periodicity and the global behavior of solutions of the following four rational difference equations

$$
\begin{aligned}
x_{n+1} & =\frac{ \pm 1}{x_{n}\left(x_{n-1} \pm 1\right)-1} \\
x_{n+1} & =\frac{ \pm 1}{x_{n}\left(x_{n-1} \mp 1\right)+1} .
\end{aligned}
$$

2010 Mathematics Subject Classification. 39A10, 39A30

Keywords: difference equations, form of solutions, equilibrium point, tribonacci number, global asymptotic stability.

\section{Introduction}

Difference equation or discrete dynamical system is a diverse field which impact almost every branch of pure and applied mathematics. Lately, there has been great interest in the study of solving difference equations and systems of difference equations, see [1-10]. In these studies, the authors deal with the closed-form, stability,periodicity, boundeness and asymptotic behavior of solutions of nonlinear difference equations and systems of difference equations. There are many recent investigations and interest in the field which difference equations have been studied by several authors, as in the examples given below:

In [1], Tollu et al. considered the following difference equations

$$
x_{n+1}=\frac{1}{1+x_{n}}, \quad y_{n+1}=\frac{1}{-1+y_{n}}, \quad n=0,1, \ldots,
$$

such that their solutions are associated with Fibonacci numbers.

In [5], Halim and Bayram investigated the solutions, stability character, and asymptotic behavior of the difference equation

$$
x_{n+1}=\frac{\alpha}{\beta+\gamma x_{n-k}}, n \in \mathbb{N}_{0},
$$


where the initial conditions $x_{-k}, x_{-k+1}, \ldots, x_{0}$ are nonzero real numbers, such that its solutions are associated to Horadam numbers, which are generalized Fibonacci numbers.

Then, in [6] Halim considered the system of difference equations

$$
x_{n+1}=\frac{1}{1+y_{n-2}}, \quad y_{n+1}=\frac{1}{1+x_{n-2}}, \quad n=0,1, \ldots,
$$

such that their solutions are associated with Fibonacci numbers, where $\mathbb{N}_{0}=\mathbb{N} \cup\{0\}$ and the initial conditions $x_{-2}, x_{-1}, x_{0}, y_{-2}, y_{-1}$, and $y_{0}$ are real numbers.

In [7], Halim and Rabago studied the systems of difference equaions

$$
x_{n+1}=\frac{1}{ \pm 1 \pm y_{n-k}}, \quad y_{n+1}=\frac{1}{ \pm 1 \pm x_{n-k}}, \quad n, k \in \mathbb{N}_{0}
$$

where the initial conditions $x_{-k}, x_{-k+1}, \ldots, x_{0}, y_{-k}, y_{-k+1}, \ldots, y_{0}$ are nonzero real numbers.

Then, in [8], the authors studied the rational difference equation

$$
x_{n+1}=\frac{\alpha x_{n-1}+\beta}{\gamma x_{n} x_{n-1}}, n \in \mathbb{N}_{0},
$$

where $\mathbb{N}_{0}=\mathbb{N} \cup\{0\}, \alpha, \beta, \gamma \in \mathbb{R}^{+}$and the initial conditions nonzero real numbers and also investigated the two-dimensional case of the this equation given by

$$
x_{n+1}=\frac{\alpha x_{n-1}+\beta}{\gamma y_{n} x_{n-1}}, y_{n+1}=\frac{\alpha y_{n-1}+\beta}{\gamma x_{n} y_{n-1}}, n \in \mathbb{N}_{0} .
$$

Also, the solutions of Eq. (5) and system of (6) are associated to generalized Padovan numbers.

As far as we examine, there is no paper dealing with the following difference equations. Hence, in this study, we study the following four difference equations

$$
\begin{aligned}
& x_{n+1}=\frac{1}{x_{n}\left(x_{n-1}+1\right)-1}, \quad n=0,1, \ldots, \\
& x_{n+1}=\frac{-1}{x_{n}\left(x_{n-1}-1\right)-1}, \quad n=0,1, \ldots, \\
& x_{n+1}=\frac{1}{x_{n}\left(x_{n-1}-1\right)+1}, \quad n=0,1, \ldots, \\
& x_{n+1}=\frac{-1}{x_{n}\left(x_{n-1}+1\right)+1}, \quad n=0,1, \ldots
\end{aligned}
$$

\section{Preliminaries}

Let $I$ be some interval of real numbers and let $f: I^{k+1} \rightarrow I$ be a continuously differentiable function. A difference equation of order $(k+1)$ is an equation of the form

$$
x_{n+1}=f\left(x_{n}, x_{n-1}, \ldots, x_{n-k}\right), \quad n=0,1, \ldots
$$

A solution of Eq.(11) is a sequence $\left\{x_{n}\right\}_{n=-k}^{\infty}$ that satisfies Eq.(11) for all $n \geq-k$. 
ON THE SOLUTIONS OF FOUR SECOND-ORDER NONLINEAR DIFFERENCE EQUATIONS3

Definition 1. A solution of Eq.(11) that is constant for all $n \geq-k$ is called an equilibrium solution of Eq.(11). If

$$
x_{n}=\bar{x}, \text { for all } n \geq-k
$$

is an equilibrium solution of Eq.(11), then $\bar{x}$ is called an equilibrium point, or simply an equilibrium of Eq.(11)..

Definition 2 (Stability). Let $\bar{x}$ an equilibrium point of $E q(11)$.

(a): An equilibrium point $\bar{x}$ of Eq.(11) is called locally stable if, for every $\varepsilon>0$; there exists $\delta>0$ such that if $\left\{x_{n}\right\}_{n=-k}^{\infty}$ is a solution of Eq.(11) with

$$
\left|x_{-k}-\bar{x}\right|+\left|x_{1-k}-\bar{x}\right|+\ldots+\left|x_{0}-\bar{x}\right|<\delta,
$$

then

$$
\left|x_{n}-\bar{x}\right|<\varepsilon, \text { for all } n \geq-k .
$$

(b): An equilibrium point $\bar{x}$ of Eq.(11) is called locally asymptotically stable if, it is locally stable, and if in addition there exists $\gamma>0$ such that if $\left\{x_{n}\right\}_{n=-k}^{\infty}$ is a solution of Eq.(11) with

$$
\left|x_{-k}-\bar{x}\right|+\left|x_{-k+1}-\bar{x}\right|+\ldots+\left|x_{0}-\bar{x}\right|<\gamma,
$$

then we have

$$
\lim _{n \rightarrow \infty} x_{n}=\bar{x}
$$

(c): An equilibrium point $\bar{x}$ of Eq.(11) is called a global attractor if, for every solution $\left\{x_{n}\right\}_{n=-k}^{\infty}$ of Eq.(11), we have

$$
\lim _{n \rightarrow \infty} x_{n}=\bar{x}
$$

(d): An equilibrium point $\bar{x}$ of Eq.(11) is called globally asymptotically stable if it is locally stable, and a global attractor.

(e): An equilibrium point $\bar{x}$ of Eq.(11) is called unstable if it is not locally stable.Suppose that the function $f$ is continuously differentiable in some open neighborhood of an equilibrium point $\bar{x}$. Let

$$
q_{i}=\frac{\partial f}{\partial u_{i}}(\bar{x}, \bar{x}, \ldots, \bar{x}), \text { for } i=0,1, \ldots, k
$$

denote the partial derivative of $f\left(u_{0}, u_{1}, \ldots, u_{k}\right)$ with respect to $u_{i}$ evaluated at the equilibrium point $\bar{x}$ of Eq.(11).

DeFinition 3. The equation

$$
y_{n+1}=q_{0} y_{n}+q_{1} y_{n-1}+\ldots+q_{k} y_{n-k}, n=0,1, \ldots
$$

is called the linearized equation of Eq.(11) about the equilibrium point $\bar{x}$, and the equation

$$
\lambda^{k+1}-q_{0} \lambda^{k}-\ldots-q_{k-1} \lambda-q_{k}=0
$$

is called the characteristic equation of Eq.(12) about $\bar{x}$.

Theorem 4 (The Linearized Stability Theorem). Assume that the function $f$ is a continuously differentiable function defined on some open neighborhood of an equilibrium point $\bar{x}$. Then the following statements are true:

(a): When all the roots of characteristic equation (13) have absolute value less than one, then the equilibrium point $\bar{x}$ of Eq.(11) is locally asymptotically stable. 
(b): If at least one root of characteristic equation (13) has absolute value greater than one, then the equilibrium point $\bar{x}$ of Eq.(11) is unstable.

The equilibrium point $\bar{x}$ of Eq.(11) is called hyperbolic if no root of characteristic equation (13) has absolute value equal to one. If there exists a root of characteristic equation (13) with absolute value equal to one, then the equilibrium $\bar{x}$ is called nonhyperbolic.

An equilibrium point $\bar{x}$ of Eq.(11) is called a repeller if all roots of characteristic equation (13) have absolute value greater than one.

An equilibrium point $\bar{x}$ of Eq.(11) is called a saddle if one of the roots of characteristic equation (13) is greater and another is less than one in absolute value.

\section{Main Results}

In this section, we present our main results for the above mentioned difference equations. Our aim is to investigate the general solutions in explicit form of the above mentioned difference equations.and the asymptotic behavior of solutions of these difference equations.

\subsection{The Difference Equation (7).}

Theorem 5. Let $\left\{x_{n}\right\}_{n=-1}^{\infty}$ be a solution of Eq.(7). Then, for $n=0,1,2, \ldots$, the forms of solutions $\left\{x_{n}\right\}_{n=-1}^{\infty}$ are given by

$$
\begin{aligned}
x_{2 n-1} & =\frac{(1-n) x_{-1} x_{0}+n}{n x_{-1} x_{0}+x_{0}-n} \\
x_{2 n} & =\frac{n x_{-1} x_{0}+x_{0}-n}{-n x_{-1} x_{0}+(n+1)}
\end{aligned}
$$

where the initial conditions $x_{-1}, x_{0} \in \mathbb{R}-F$, with $F$ is the forbidden set of Eq.(7) given by

$$
F=\bigcup_{n=1}^{\infty}\left\{\left(x_{-1}, x_{0}\right): x_{-1}=-\frac{n+1}{n}, x_{0}=-1\right\} .
$$

Proof. For $n=0$ the result holds. Assume that $n>0$ and that our assumption holds for $n-1$. That is,

$$
x_{2 n-3}=\frac{(2-n) x_{-1} x_{0}+n-1}{(n-1) x_{-1} x_{0}+x_{0}-(n-1)}
$$

and

$$
x_{2 n-2}=\frac{(n-1) x_{-1} x_{0}+x_{0}-(n-1)}{(1-n) x_{-1} x_{0}+n} .
$$

From this and from Eq.(7), it follows that

$$
\begin{aligned}
x_{2 n-1} & =\frac{1}{x_{2 n-2}\left(x_{2 n-3}+1\right)-1} \\
& =\frac{1}{\frac{(n-1) x_{-1} x_{0}+x_{0}-(n-1)}{(1-n) x_{-1} x_{0}+n}\left(\frac{(2-n) x_{-1} x_{0}+n-1}{(n-1) x_{-1} x_{0}+x_{0}-(n-1)}+1\right)-1} \\
& =\frac{(1-n) x_{-1} x_{0}+n}{n x_{-1} x_{0}+x_{0}-n} .
\end{aligned}
$$


Hence, similarly, we obtain

$$
\begin{aligned}
x_{2 n} & =\frac{1}{x_{2 n-1}\left(x_{2 n-2}+1\right)-1} \\
& =\frac{1}{\frac{(1-n) x_{-1} x_{0}+n}{n x_{-1} x_{0}+x_{0}-n}\left(\frac{(n-1) x_{-1} x_{0}+x_{0}-(n-1)}{(1-n) x_{-1} x_{0}+n}+1\right)-1} \\
& =\frac{n x_{-1} x_{0}+x_{0}-n}{-n x_{-1} x_{0}+n+1} .
\end{aligned}
$$

THeOREM 6. The following statements are true.

(i): The equilibrium points of Eq.(7) are $\bar{x}_{1}=1$ and $\bar{x}_{2}=-1$.

(ii): The positive equilibrium point of Eq.(7), $\bar{x}_{1}=1$, is nonhyperbolic point.

(iii): The negative equilibrium point of Eq.(7), $\bar{x}_{2}=-1$, is nonhyperbolic point.

\section{Proof.}

(i): Equilibrium points of Eq.(7) satisfy the equation

$$
\bar{x}=\frac{1}{\bar{x}(\bar{x}+1)-1} .
$$

After simplification, we have the following cubic equation

$$
\bar{x}^{3}+\bar{x}^{2}-\bar{x}-1=0 \text {. }
$$

The roots of the cubic equation (16) are $-1,-1,1$. Therefore, Eq.(7) has two equilibra, one positive and one negative, such that

$$
\bar{x}_{1}=1, \bar{x}_{2}=-1 \text {. }
$$

(ii): Now, let $I=(0, \infty)$ and consider the function

$$
f: I^{2} \rightarrow I
$$

defined by

$$
f(x, y)=\frac{1}{x(y+1)-1} .
$$

Then, it follows that

$$
\begin{aligned}
& \frac{\partial f(x, y)}{\partial x}=\frac{-(y+1)}{(x(y+1)-1)^{2}}, \\
& \frac{\partial f(x, y)}{\partial y}=\frac{-x}{(x(y+1)-1)^{2}} .
\end{aligned}
$$

Therefore, the linearized equation of Eq.(7) about the equilibrium point $\bar{x}_{1}=1$ is

$$
z_{n+1}=p z_{n}+q z_{n-1}
$$

where

$$
\begin{aligned}
p & =\frac{\partial f\left(\bar{x}_{1}, \bar{x}_{1}\right)}{\partial x}=-2, \\
q & =\frac{\partial f\left(\bar{x}_{1}, \bar{x}_{1}\right)}{\partial y}=-1,
\end{aligned}
$$

and the corresponding characteristic equation is

$$
\lambda^{2}+2 \lambda+1=0 .
$$


Therefore, from Theorem (4), it is clearly seen that

$$
\lambda_{1,2}=-1
$$

and

$$
\left|\lambda_{1}\right|=\left|\lambda_{2}\right|=1
$$

So, $\bar{x}_{1}$ is nonhyperbolic point.

(iii): Similarly, from (17), The linearized equation of Eq.(7) about the equilibrium point $\bar{x}_{2}=-1$ is

$$
z_{n+1}=p z_{n}+q z_{n-1},
$$

where

$$
\begin{aligned}
& p=\frac{\partial f\left(\bar{x}_{2}, \bar{x}_{2}\right)}{\partial x}=0, \\
& q=\frac{\partial f\left(\bar{x}_{2}, \bar{x}_{2}\right)}{\partial y}=1,
\end{aligned}
$$

and its characteristic equation is

$$
\lambda^{2}-1=0 .
$$

Thus, it follows that

$$
\lambda_{1,2}= \pm 1
$$

and

$$
\left|\lambda_{1}\right|=\left|\lambda_{2}\right|=1 .
$$

So, $\bar{x}_{2}$ is nonhyperbolic point.

Theorem 7. Let $\left\{x_{n}\right\}_{n=-1}^{\infty}$ be a solution of Eq.(7). Then, the negative equilibrium point of Eq.(7), $\bar{x}_{2}$, is a global attractor.

Proof. From Theorem (5), we have

$$
\begin{aligned}
\lim _{n \rightarrow \infty} x_{2 n-1} & =\lim _{n \rightarrow \infty} \frac{(1-n) x_{-1} x_{0}+n}{n x_{-1} x_{0}+x_{0}-n} \\
& =\lim _{n \rightarrow \infty} \frac{(1-n)\left(x_{-1} x_{0}+\frac{n}{1-n}\right)}{n\left(x_{-1} x_{0}+\frac{x_{0}}{n}-1\right)} \\
& =\lim _{n \rightarrow \infty} \frac{(1-n)\left(x_{-1} x_{0}-1+\frac{1}{1-n}\right)}{n\left(x_{-1} x_{0}+\frac{x_{0}}{n}-1\right)} \\
& =-1,
\end{aligned}
$$

and

$$
\begin{aligned}
\lim _{n \rightarrow \infty} x_{2 n} & =\lim _{n \rightarrow \infty} \frac{n x_{-1} x_{0}+x_{0}-n}{-n x_{-1} x_{0}+(n+1)} \\
& =\lim _{n \rightarrow \infty} \frac{n\left(x_{-1} x_{0}+\frac{x_{0}}{n+1}-1\right)}{-n\left(x_{-1} x_{0}-1-\frac{1}{n}\right)} \\
& =-1 .
\end{aligned}
$$

Hereby, it implies

$$
\lim _{n \rightarrow \infty} x_{n}=-1 .
$$


ON THE SOLUTIONS OF FOUR SECOND-ORDER NONLINEAR DIFFERENCE EQUATIONS7

\subsection{The Difference Equation (8).}

TheOREM 8. Let $\left\{x_{n}\right\}_{n=-1}^{\infty}$ be a solution of Eq.(8). Then, for $n=0,1,2, \ldots$, the forms of solutions $\left\{x_{n}\right\}_{n=-1}^{\infty}$ are given by

$$
\begin{aligned}
x_{2 n-1} & =\frac{-\left((1-n) x_{-1} x_{0}+n\right)}{n x_{-1} x_{0}-x_{0}-n} \\
x_{2 n} & =\frac{-\left(n x_{-1} x_{0}-x_{0}-n\right)}{-n x_{-1} x_{0}+n+1}
\end{aligned}
$$

where the initial conditions $x_{-1}, x_{0} \in \mathbb{R}-F$, with $F$ is the forbidden set of Eq.(8) given by

$$
F=\bigcup_{n=1}^{\infty}\left\{\left(x_{-1}, x_{0}\right): x_{-1}=\frac{n+1}{n}, x_{0}=1\right\} .
$$

Proof. For $n=0$ the result holds. Assume that $n>0$ and that our assumption holds for $n-1$. That is,

$$
x_{2 n-3}=\frac{-\left((2-n) x_{-1} x_{0}+n-1\right)}{(n-1) x_{-1} x_{0}-x_{0}-(n-1)}
$$

and

$$
x_{2 n-2}=\frac{-\left((n-1) x_{-1} x_{0}-x_{0}-(n-1)\right)}{-(n-1) x_{-1} x_{0}+n} .
$$

From this and from Eq.(8), it follows that

$$
\begin{aligned}
x_{2 n-1} & =\frac{-1}{x_{2 n-2}\left(x_{2 n-3}-1\right)-1} \\
& =\frac{-1}{\frac{-\left((n-1) x_{-1} x_{0}-x_{0}-(n-1)\right)}{-(n-1) x_{-1} x_{0}+n}\left(\frac{-\left((2-n) x_{-1} x_{0}+n-1\right)}{(n-1) x_{-1} x_{0}-x_{0}-(n-1)}-1\right)-1} \\
& =\frac{-\left((1-n) x_{-1} x_{0}+n\right)}{n x_{-1} x_{0}-x_{0}-n} .
\end{aligned}
$$

Hence, similarly, we obtain

$$
\begin{aligned}
x_{2 n} & =\frac{-1}{x_{2 n-1}\left(x_{2 n-2}-1\right)-1} \\
& =\frac{-1}{\frac{-\left((1-n) x_{-1} x_{0}+n\right)}{n x_{-1} x_{0}-x_{0}-n}\left(\frac{-\left((n-1) x_{-1} x_{0}-x_{0}-(n-1)\right)}{-(n-1) x_{-1} x_{0}+n}-1\right)-1} \\
& =\frac{-\left(n x_{-1} x_{0}-x_{0}-n\right)}{-n x_{-1} x_{0}+n+1} .
\end{aligned}
$$

THEOREM 9. The following statements are true.

(i): The equilibrium points of Eq.(8) are $\bar{x}_{1}=1$ and $\bar{x}_{2}=-1$.

(ii): The positive equilibrium point of $E q .(8), \bar{x}_{1}=1$, is nonhyperbolic point.

(iii): The negative equilibrium point of Eq.(8), $\bar{x}_{2}=-1$, is nonhyperbolic point.

\section{Proof.}

(i): Equilibrium points of Eq.(8) satisfy the equation

$$
\bar{x}=\frac{-1}{\bar{x}(\bar{x}-1)-1} .
$$


After simplification, we have the following cubic equation

$$
\bar{x}^{3}-\bar{x}^{2}-\bar{x}+1=0 \text {. }
$$

The roots of the cubic equation (20) are $-1,1,1$. Therefore, Eq.(8) has two equilibra, one positive and one negative, such that

$$
\bar{x}_{1}=1, \bar{x}_{2}=-1 \text {. }
$$

(ii): Now, let $I=(0, \infty)$ and consider the function

$$
f: I^{2} \rightarrow I
$$

defined by

$$
f(x, y)=\frac{-1}{x(y-1)-1} .
$$

Then, it follows that

$$
\begin{aligned}
& \frac{\partial f(x, y)}{\partial x}=\frac{(y-1)}{(x(y-1)-1)^{2}}, \\
& \frac{\partial f(x, y)}{\partial y}=\frac{x}{(x(y-1)-1)^{2}} .
\end{aligned}
$$

Therefore, the linearized equation of Eq.(8) about the equilibrium point $\bar{x}_{1}=1$ is

where

$$
z_{n+1}=p z_{n}+q z_{n-1},
$$

$$
\begin{aligned}
p & =\frac{\partial f\left(\bar{x}_{1}, \bar{x}_{1}\right)}{\partial x}=0, \\
q & =\frac{\partial f\left(\bar{x}_{1}, \bar{x}_{1}\right)}{\partial y}=1,
\end{aligned}
$$

and the corresponding characteristic equation is

$$
\lambda^{2}-1=0 .
$$

Therefore, from Theorem (4), it is clearly seen that

$$
\lambda_{1,2}= \pm 1
$$

and

$$
\left|\lambda_{1}\right|=\left|\lambda_{2}\right|=1
$$

So, $\bar{x}_{1}$ is nonhyperbolic point.

(iii): Similarly, from (21), The linearized equation of Eq.(8) about the equilibrium point $\bar{x}_{2}=-1$ is

$$
z_{n+1}=p z_{n}+q z_{n-1},
$$

where

$$
\begin{aligned}
& p=\frac{\partial f\left(\bar{x}_{2}, \bar{x}_{2}\right)}{\partial x}=-2, \\
& q=\frac{\partial f\left(\bar{x}_{2}, \bar{x}_{2}\right)}{\partial y}=-1,
\end{aligned}
$$

and its characteristic equation is

$$
\lambda^{2}+2 \lambda+1=0
$$


ON THE SOLUTIONS OF FOUR SECOND-ORDER NONLINEAR DIFFERENCE EQUATIONS9

Thus, it follows that

$$
\lambda_{1,2}=-1
$$

and

$$
\left|\lambda_{1}\right|=\left|\lambda_{2}\right|=1
$$

So, $\bar{x}_{2}$ is nonhyperbolic point.

ThEOREM 10. Let $\left\{x_{n}\right\}_{n=-1}^{\infty}$ be a solution of Eq.(8). Then, the positive equilibrium point of Eq.(8), $\bar{x}_{1}$, is a global attractor.

Proof. From Theorem (8), we obtain

$$
\begin{aligned}
\lim _{n \rightarrow \infty} x_{2 n-1} & =\lim _{n \rightarrow \infty} \frac{-\left((1-n) x_{-1} x_{0}+n\right)}{n x_{-1} x_{0}-x_{0}-n} \\
& =\lim _{n \rightarrow \infty} \frac{(n-1)\left(x_{-1} x_{0}+\frac{n}{1-n}\right)}{n\left(x_{-1} x_{0}-\frac{x_{0}}{n}-1\right)} \\
& =\lim _{n \rightarrow \infty} \frac{(n-1)\left(x_{-1} x_{0}-1+\frac{1}{1-n}\right)}{n\left(x_{-1} x_{0}-\frac{x_{0}}{n}-1\right)} \\
& =1,
\end{aligned}
$$

and

$$
\begin{aligned}
\lim _{n \rightarrow \infty} x_{2 n} & =\lim _{n \rightarrow \infty} \frac{-\left(n x_{-1} x_{0}-x_{0}-n\right)}{-n x_{-1} x_{0}+n+1} \\
& =\lim _{n \rightarrow \infty} \frac{-n\left(x_{-1} x_{0}-\frac{x_{0}}{n}-1\right)}{-n\left(x_{-1} x_{0}-1-\frac{1}{n}\right)} \\
& =1 .
\end{aligned}
$$

Herewith, it implies

$$
\lim _{n \rightarrow \infty} x_{n}=1
$$

So, the proof is complete.

\subsection{The Difference Equation (9).}

LEMma 11. Let $\left\{x_{n}\right\}_{n=-1}^{\infty}$ be a solution of Eq.(9). Then, $\left\{x_{n}\right\}_{n=-1}^{\infty}$ is periodic with period four.

Proof. From Eq.(9),

$$
\begin{aligned}
x_{n+4} & =\frac{1}{x_{n+3}\left(x_{n+2}-1\right)+1} \\
& =\frac{1}{\left(\frac{1}{x_{n+2}\left(x_{n+1}-1\right)+1}\right)\left(\frac{1}{x_{n+1}\left(x_{n}-1\right)+1}-1\right)+1}
\end{aligned}
$$




$$
\begin{aligned}
& =\frac{1}{\left(\frac{1}{\left(\frac{1}{x_{n+1}\left(x_{n}-1\right)+1}\right)\left(\frac{1}{x_{n}\left(x_{n-1}-1\right)+1}-1\right)+1}\right)\left(\frac{1}{\left(\frac{1}{x_{n}\left(x_{n-1}-1\right)+1}\right)\left(x_{n}-1\right)+1}-1\right)+1} \\
& =\frac{1}{\left(\frac{1}{\left(\frac{1}{\overline{x_{n}\left(x_{n-1}-1\right)+1}\left(x_{n}-1\right)+1}\right)\left(\frac{x_{n}\left(1-x_{n-1}\right)}{x_{n} x_{n-1}-x_{n}+1}\right)+1}\right)\left(\frac{1-x_{n}}{x_{n} x_{n-1}}\right)+1} \\
& =\frac{1}{x_{n-1}\left(\frac{1-x_{n}}{x_{n} x_{n-1}}\right)+1} \\
& =x_{n} \text {. }
\end{aligned}
$$

Hence, the result holds.

Theorem 12. Let $\left\{x_{n}\right\}_{n=-1}^{\infty}$ be a solution of Eq.(9). Then, for $n=1,2, \ldots$,

$$
\begin{aligned}
x_{4 n-3} & =\frac{1}{x_{-1} x_{0}-x_{0}+1} \\
x_{4 n-2} & =\frac{x_{-1} x_{0}-x_{0}+1}{x_{-1} x_{0}} \\
x_{4 n-1} & =x_{-1} \\
x_{4 n} & =x_{0}
\end{aligned}
$$

where the initial conditions $x_{-1}, x_{0} \in \mathbb{R}-F$, with $F$ is the forbidden set of Eq.(9) given by

$$
F=\left\{\left(x_{-1}, x_{0}\right): x_{-1} x_{0}=0, x_{-1}=\frac{x_{0}-1}{x_{0}}\right\} .
$$

Proof. From (9), for $n=0$, the result holds. Suppose that $n>0$ and that our assumption holds for $n-1$. That is,

$$
\begin{aligned}
x_{4 n-7} & =\frac{1}{x_{-1} x_{0}-x_{0}+1}, \\
x_{4 n-6} & =\frac{x_{-1} x_{0}-x_{0}+1}{x_{-1} x_{0}}, \\
x_{4 n-5} & =x_{-1}, \\
x_{4 n-4} & =x_{0} .
\end{aligned}
$$

Now, from Eq.(9), it follows that

$$
x_{4 n-3}=\frac{1}{x_{4 n-4}\left(x_{4 n-5}-1\right)+1}=\frac{1}{x_{-1} x_{0}-x_{0}+1} .
$$

From this and from Eq.(9), it follows that

$$
x_{4 n-2}=\frac{1}{x_{4 n-3}\left(x_{4 n-4}-1\right)+1}=\frac{1}{\frac{1}{x_{-1} x_{0}-x_{0}+1}\left(x_{0}-1\right)+1}=\frac{x_{-1} x_{0}-x_{0}+1}{x_{-1} x_{0}} .
$$

Again from Eq.(9), we get

$$
x_{4 n-1}=\frac{1}{x_{4 n-2}\left(x_{4 n-3}-1\right)+1}=\frac{1}{\frac{x_{-1} x_{0}-x_{0}+1}{x_{-1} x_{0}}\left(\frac{1}{x_{-1} x_{0}-x_{0}+1}-1\right)+1}=\frac{x_{-1} x_{0}}{x_{0}}=x_{-1} .
$$


ON THE SOLUTIONS OF FOUR SECOND-ORDER NONLINEAR DIFFERENCE EQUATION\$1

Similarly, from Eq.(9), we have

$$
\begin{aligned}
x_{4 n-4} & =\frac{1}{x_{4 n-1}\left(x_{4 n-2}-1\right)+1}=\frac{1}{x_{-1}\left(\frac{x_{-1} x_{0}-x_{0}+1}{x_{-1} x_{0}}-1\right)+1} \\
& =\frac{1}{x_{-1}-1+\frac{1}{x_{0}}-x_{-1}+1}=x_{0} .
\end{aligned}
$$

Thus, the proof is complete.

Theorem 13. Eq.(9) has unique positive equilibrium point $\bar{x}=1$ and 1 is nonhyperbolic point.

Proof. Equilibrium point of Eq.(9) satisfy the equation

$$
\bar{x}=\frac{1}{\bar{x}(\bar{x}-1)+1} \text {. }
$$

After simplification, we have the following cubic equation

$$
\bar{x}^{3}-\bar{x}^{2}+\bar{x}-1=0 .
$$

The roots of the cubic equation (23) are $-i, i, 1$. Therefore, the unique positive equilibrium point of Eq.(9) is $\bar{x}=1$.

Now, we prove that the equilibrium point of Eq.(9) is nonhyperbolic.

Let $I=(0, \infty)$ and consider the function

$$
f: I^{2} \rightarrow I
$$

defined by

$$
f(x, y)=\frac{1}{x(y-1)+1} .
$$

The linearized equation of Eq.(9) about the equilibrium point $\bar{x}=1$ is

$$
z_{n+1}=p z_{n}+q z_{n-1}
$$

where

$$
\begin{aligned}
p & =\frac{\partial f(\bar{x}, \bar{x})}{\partial x}=0 \\
q & =\frac{\partial f(\bar{x}, \bar{x})}{\partial y}=-1
\end{aligned}
$$

and the corresponding characteristic equation is

$$
\lambda^{2}+1=0 .
$$

Therefore, from Theorem (4), it is clearly seen that

$$
\lambda_{1,2}= \pm i
$$

and

$$
\left|\lambda_{1}\right|=\left|\lambda_{2}\right|=1
$$

So, this completes the proof. 


\subsection{The Difference Equation (10).}

Lemma 14. Let $\left\{x_{n}\right\}_{n=-1}^{\infty}$ be a solution of Eq.(10). Then, $\left\{x_{n}\right\}_{n=-1}^{\infty}$ is periodic with periods four.

Proof. From Eq.(10),

$$
\begin{aligned}
x_{n+4} & =\frac{-1}{x_{n+3}\left(x_{n+2}+1\right)+1} \\
& =\frac{-1}{\left(\frac{-1}{x_{n+2}\left(x_{n+1}+1\right)+1}\right)\left(\frac{-1}{x_{n+1}\left(x_{n}+1\right)+1}+1\right)+1} \\
& =\frac{-1}{\left(\frac{-1}{\left(\frac{-1}{x_{n+1}\left(x_{n}+1\right)+1}\right)\left(\frac{-1}{x_{n}\left(x_{n-1}+1\right)+1}+1\right)+1}\right)\left(\frac{-1}{\left(\frac{-1}{x_{n}\left(x_{n-1}+1\right)+1}\right)\left(x_{n}+1\right)+1}+1\right)+1} \\
& \left.=\frac{-1}{\left(\frac{-1}{\left(\frac{-1}{x_{n}\left(x_{n-1}+1\right)+1}\left(x_{n}+1\right)+1\right.}\right)\left(\frac{x_{n}\left(x_{n-1}+1\right)}{x_{n} x_{n-1}+x_{n}+1}\right)+1}\right)\left(-\frac{x_{n}+1}{x_{n} x_{n-1}}\right)+1 \\
& =\frac{-1}{x_{n-1}\left(-\frac{x_{n}+1}{x_{n} x_{n-1}}\right)+1} \\
& =x_{n} .
\end{aligned}
$$

Hence, the result holds.

Theorem 15. Let $\left\{x_{n}\right\}_{n=-1}^{\infty}$ be a solution of Eq.(10). Then, for $n=1,2, \ldots$,

$$
\begin{aligned}
x_{4 n-3} & =\frac{-1}{x_{-1} x_{0}+x_{0}+1} \\
x_{4 n-2} & =\frac{-\left(x_{-1} x_{0}+x_{0}+1\right)}{x_{-1} x_{0}} \\
x_{4 n-1} & =x_{-1} \\
x_{4 n} & =x_{0}
\end{aligned}
$$

where the initial conditions $x_{-1}, x_{0} \in \mathbb{R}-F$, with $F$ is the forbidden set of Eq.(10) given by

$$
F=\left\{\left(x_{-1}, x_{0}\right): x_{-1} x_{0}=0, x_{-1}=\frac{-\left(x_{0}+1\right)}{x_{0}}\right\} .
$$

Proof. From (10), for $n=0$, the result holds. Suppose that $n>0$ and that our assumption holds for $n-1$. That is,

$$
\begin{aligned}
x_{4 n-7} & =\frac{-1}{x_{-1} x_{0}+x_{0}+1} \\
x_{4 n-6} & =\frac{-\left(x_{-1} x_{0}+x_{0}+1\right)}{x_{-1} x_{0}} \\
x_{4 n-5} & =x_{-1} \\
x_{4 n-4} & =x_{0} .
\end{aligned}
$$


ON THE SOLUTIONS OF FOUR SECOND-ORDER NONLINEAR DIFFERENCE EQUATION\$3

Now, from Eq.(10), it follows that

$$
x_{4 n-3}=\frac{-1}{x_{4 n-4}\left(x_{4 n-5}+1\right)+1}=\frac{-1}{x_{-1} x_{0}+x_{0}+1} .
$$

From this and from Eq.(10), it follows that

$$
x_{4 n-2}=\frac{-1}{x_{4 n-3}\left(x_{4 n-4}+1\right)+1}=\frac{-1}{\frac{-1}{x_{-1} x_{0}+x_{0}+1}\left(x_{0}+1\right)+1}=\frac{-\left(x_{-1} x_{0}+x_{0}+1\right)}{x_{-1} x_{0}} .
$$

Again from Eq.(10), we get

$$
x_{4 n-1}=\frac{-1}{x_{4 n-2}\left(x_{4 n-3}+1\right)+1}=\frac{-1}{\frac{-\left(x_{-1} x_{0}+x_{0}+1\right)}{x_{-1} x_{0}}\left(\frac{-1}{x_{-1} x_{0}+x_{0}+1}+1\right)+1}=\frac{-x_{-1} x_{0}}{-x_{0}}=x_{-1} .
$$

Similarly, from Eq.(10), we have

$$
\begin{aligned}
x_{4 n} & =\frac{-1}{x_{4 n-1}\left(x_{4 n-2}+1\right)+1}=\frac{-1}{x_{-1}\left(\frac{-\left(x_{-1} x_{0}+x_{0}+1\right)}{x_{-1} x_{0}}+1\right)+1} \\
& =\frac{-1}{-x_{-1}-1-\frac{1}{x_{0}}+x_{-1}+1}=x_{0} .
\end{aligned}
$$

Thus, the proof is complete.

Theorem 16. Eq.(10) has unique positive equilibrium point $\bar{x}=1$ and the equilibrium point 1 is locally assymptotically stable.

Proof. Equilibrium point of Eq.(10) satisfy the equation

$$
\bar{x}=\frac{-1}{\bar{x}(\bar{x}+1)+1} .
$$

After simplification, we have the following cubic equation

$$
\bar{x}^{3}+\bar{x}^{2}+\bar{x}+1=0 \text {. }
$$

The roots of the cubic equation $(25)$ are $-i, i, 1$. Therefore, the unique positive equilibrium point of Eq.(10) is $\bar{x}=1$.

Now, we demonstrate that the equilibrium point of Eq.(10) is nonhyperbolic.

Let $I=(0, \infty)$ and consider the function

$$
f: I^{2} \rightarrow I
$$

defined by

$$
f(x, y)=\frac{-1}{x(y+1)+1} .
$$

The linearized equation of Eq.(10) about the equilibrium point $\bar{x}=1$ is

$$
z_{n+1}=p z_{n}+q z_{n-1}
$$

where

$$
\begin{aligned}
p & =\frac{\partial f(\bar{x}, \bar{x})}{\partial x}=\frac{2}{9} \\
q & =\frac{\partial f(\bar{x}, \bar{x})}{\partial y}=\frac{1}{9}
\end{aligned}
$$

and the corresponding characteristic equation is

$$
\lambda^{2}-\frac{2}{9} \lambda-\frac{1}{9}=0 .
$$


Therefore, from Theorem (4), it is clearly seen that

$$
\lambda_{1,2}=\frac{1}{9} \pm \frac{1}{9} \sqrt{10}
$$

and

Thus, the proof is complete.

$$
\left|\lambda_{1,2}\right|<1
$$

\section{References}

[1] Tollu, DT, Yazlik, Y, Taskara, N, On the solutions of two special types of Riccati difference equation via Fibonacci numbers. Advances in Difference Equations, 2013:174 (2013)

[2] Yazlik, Y, Tollu, DT, Taskara, N, On the Solutions of Difference Equation Systems with Padovan Numbers. Applied Mathematics, 4:15-20 (2013)

[3] Tollu, DT, Yazlik, Y, Taskara, N, On fourteen solvable systems of difference equations. Applied Mathematics and Computation, 233: 310-319 (2014)

[4] Halim, Y, Global Character of Systems of Rational Difference Equations. Electronic Journal of Mathematical Analysis and Applications, 3(1): 204-214 (2015)

[5] Halim, Y, Bayram, M, On the solutions of a higher-order difference equation in terms of generalized Fibonacci sequences. Mathematical Methods in the Applied Sciences, 39: 2974$2982(2016)$

[6] Halim, Y, A System of Difference Equations with Solutions Associated to Fibonacci Numbers. International Journal of Difference Equations, 11(1): 65-77 (2016)

[7] Halim, Y, Rabago, JFT, On the Some Solvable Systems of Difference Equations with Solutions Associated to Fibonacci Numbers. Electronic Journal of Mathematical Analysis and Applications, 5(1): 166-178 (2017)

[8] Halim, Y, Rabago, JFT, On the Solutions of a Second-Order Difference Equation in terms of Generalized Padovan Sequences. Mathematica Slovaca, 68(3): 625-638 (2018)

[9] Matsunaga, H, Suzuki R, Classification of global behavior of a system of rational difference equations. Applied Mathematics Letters, 85: 57-63 (2018)

[10] Öcalan, Ö, Duman, O, On Solutions of the Recursive Equations $x_{n+1}=x_{n-1}^{p} / x_{n}^{p}(p>0)$ via Fibonacci-Type Sequences. Electronic Journal of Mathematical Analysis and Applications, 7(1): 102-115 (2019) 